\title{
Criminologie
}

\section{De la prison à l'école de bienfaisance. Origines et transformations des institutions pénitentiaires pour enfants en Belgique au XIXe siècle (1840-1914)}

\section{Marie-Sylvie Dupont-Bouchat}

Volume 28, numéro 1, 1995

L'histoire de l'enfermement et des populations pénales

URI : https://id.erudit.org/iderudit/017363ar

DOI : https://doi.org/10.7202/017363ar

Aller au sommaire du numéro

Éditeur(s)

Les Presses de l'Université de Montréal

ISSN

0316-0041 (imprimé)

1492-1367 (numérique)

Découvrir la revue

Citer cet article

Dupont-Bouchat, M.-S. (1995). De la prison à l'école de bienfaisance. Origines et transformations des institutions pénitentiaires pour enfants en Belgique au XIXe siècle (1840-1914). Criminologie, 28(1), 23-53.

https://doi.org/10.7202/017363ar

\section{Résumé de l'article}

The creation and evolution of juvenile institutions in 19th century Belgium corresponds to two approaches, two eras and two reformers (Edouard Ducpétiaux and Jules LeJeune). The first fifty years are dominated by the idea of guilty children, who should be condemned and incarcerated in penitentiaries to be punished and reformed. Then, the image of children in danger who should be protected against the bad influences of their families becomes dominant. The passage from punishment to protection involves the adoption of social and psychological criteria to classify children along new categories. The scope of the institutions is being widened as their mission becomes centered on all types of children "in danger". As institutions become challenged, family placements become more popular and are intended to compensate for the weaknesses and bad influences of the natural family. The legal withdrawal of parental authority (endorsed by Parliament in 1912) is aimed at controlling families of deprived classes of society. The adoption of the medical model for juvenile delinquents becomes a reality with the opening of psychological observation centres in the Écoles de bienfaisance.
Tous droits réservés @ Les Presses de l'Université de Montréal, 1995
Ce document est protégé par la loi sur le droit d'auteur. L'utilisation des services d'Érudit (y compris la reproduction) est assujettie à sa politique d'utilisation que vous pouvez consulter en ligne.

https://apropos.erudit.org/fr/usagers/politique-dutilisation/ 


\section{DE LA PRISON À L'ÉCOLE DE BIENFAISANCE ORIGINES ET TRANSFORMATIONS DES INSTITUTIONS PÉNITENTIAIRES POUR ENFANTS EN BELGIQUE AU XIX ${ }^{\mathrm{e}}$ SIÈCLE (1840-1914) \\ (Première partie) $^{1}$ Marie-Sylvie Dupont-Bouchat ${ }^{2}$}

The creation and evolution of juvenile institutions in 19th century Belgium corresponds to two approaches, two eras and two reformers (Édouard Ducpétiaux and Jules LeJeune). The first fifty years are dominated by the idea of guilty children, who should be condemned and incarcerated in penitentiaries to be punished and reformed. Then, the image of children in danger who should be protected against the bad influences of their families becomes dominant. The passage from punishment to protection involves the adoption of social and psychological criteria to classify children along new categories. The scope of the institutions is being widened as their mission becomes centered on all types of children "in danger". As institutions become challenged, family placements become more popular and are intended to compensate for the weaknesses and bad influences of the natural family. The legal withdrawal of parental authority (endorsed by Parliament in 1912) is aimed at controlling families of deprived classes of society. The adoption of the medical model for juvenile delinquents becomes a reality with the opening of psychological observation centres in the Écoles de bienfaisance.

\section{INTRODUCTION}

Deux hommes, deux conceptions vont inspirer en Belgique au $\mathrm{XIX}^{e}$ siècle deux modèles de politique pénale vis-à-vis des mineurs délinquants. Ces modèles correspondent à deux époques : l'ère Ducpétiaux, qui s'étend au sens large de 1830 à 1887, et l'ère Lejeune, de la fin des années 1880 à 1912. La première, l' «ère Ducpétiaux», est celle de la moralisation de l'enfance coupable par la prison, le pénitencier, l'école de réforme. Ducpétiaux, inspecteur général des prisons du royaume entre 1830 et 1860 , s'inspire assez largement des modèles étrangers, la France notamment, pour créer en Belgique un double réseau d'institutions pénitentiaires et d'écoles de réforme fondé sur la distinction entre enfants coupables - c'est-à-dire condamnés par les tribunaux - et enfants malheureux - vagabonds, men-

1. La seconde partie de cet article paraîtra dans le numéro 2, vol. 28 de Criminologie, à l'automne 1995.

2. Professeur à l'Université catholique de Louvain. 
diants, orphelins et abandonnés. Les premiers seront punis, redressés et éduqués dans les pénitenciers : Saint-Hubert pour les garçons (1840), Liège (1848), puis Namur (1864) pour les filles; les seconds seront éduqués dans des écoles de réforme: Ruiselede pour les garçons et Beernem pour les filles (1848).

À partir des années 1880 , les critiques formulées contre les pratiques pénitentiaires classiques, et l'échec de celles-ci, ainsi que l'émergence d'une nouvelle pensée pénale - la défense sociale - amènent à revoir cette politique de punition des coupables fondée sur les distinctions établies par le code pénal entre condamnés et acquittés. Un nouveau modèle se dégage : il s'agira moins désormais de punir que de prévenir et éduquer. On passe de la notion d'enfance coupable à celle d'enfant en danger qu'il s'agit de protéger. Et ainsi, de la prison à l'école. Les pénitenciers et les écoles de réforme deviennent «Écoles de bienfaisance " (1890). C'est l'ère Lejeune, celle de la protection de l'enfance et du patronage.

Jules Lejeune, ministre de la Justice de 1887 à 1894, dépose en 1889 un projet de loi sur la protection de l'enfance qui aboutira, vingt-trois ans plus tard, à la loi de 1912. Entre-temps, pour tenter de faire passer ses idées, il organise en Belgique les premiers congrès internationaux sur la protection de l'enfance et le patronage (Anvers 1890, 1894 et 1898) et met sur pied un vaste réseau de comités de patronage coordonné par la Commission royale des Patronages qu'il crée en 1894.

La Belgique devient ainsi « la capitale internationale de la protection de l'enfance et du patronage " et diffuse ses idées et ses pratiques en Europe.

Ces deux modèles de politique pénale à l'égard des mineurs ne correspondent pas seulement à deux conceptions, deux visions de l'enfance coupable ou malheureuse, celles de deux hommes, Ducpétiaux et Lejeune, mais elles s'inscrivent naturellement dans deux contextes politiques et sociaux différents.

L'ère de Ducpétiaux, de la prison et de l'enfance coupable, coïncide avec le premier $X{ }^{e}{ }^{e}$ siècle, que l'on pourrait caractériser en Belgique comme l'époque du libéralisme triomphant. La révolution de 1830 , qui a doté le pays de la constitution «la plus libérale d'Europe », rassure toutes les consciences sur l'excellence du régime, son caractère "démocratique " et «égalitaire». La fiction juridique de l'égalité de tous devant la loi masque pendant cinquante ans les inégalités de fait: suffrage censitaire réservé aux nantis, infériorité juridique de la classe ouvrière, des femmes et des enfants. La stabilité politique du régime est garantie par l'alternance au pouvoir de gouvernements majoritaires catholiques ou libéraux qui ont en 
commun la même méfiance vis-à-vis de l'intervention de l'État dans le champ "privé » de la famille ou de l'entreprise ${ }^{3}$.

Par conséquent, il n'y a pas de législation sociale protectrice pour les ouvriers, pas de réglementation du travail des femmes et des enfants, pas de scolarité obligatoire. C'est le règne de la «liberté »: libre concurrence, liberté du travail, liberté du père de famille, avec pour corollaires la misère et l'ignorance de la majorité de la classe ouvrière et populaire ${ }^{4}$.

L'État-gendarme se borme à maintenir l'ordre économique et social en réprimant çà et là quelques grèves sporadiques et locales 5 .

La philanthropie et la charité privée se chargent d'apporter quelques remèdes aux abus les plus criants du capitalisme ${ }^{6}$.

Ce système fonctionne assez bien pendant une cinquantaine d'années, malgré les cris d'alarme lancés ponctuellement par quelques esprits éclairés, mais marginaux, tels Ducpétiaux, qui dénonce dès 1843 "les signes précurseurs d'une révolution nouvelle, non plus politique mais sociale, révolution du prolétariat contre la propriété ".

Celle-ci ne se produira qu'en 1886 au moment où la classe ouvrière, organisée par le tout jeune parti socialiste (créé en 1885), prend conscience de sa force et fait éclater ses revendications. Grèves spontanées, émeutes de la misère, réprimées dans le sang, en 1886 , bientôt canalisées et orientées par les socialistes vers des revendications politiques - le suffrage universel - ces grandes colères vont modifier le paysage politique et social de la Belgique à la fin du $\mathrm{XIX}^{\mathrm{e}}$ siècle $^{7}$.

Les classes dirigeantes, enfin sensibilisées aux problèmes sociaux, consentent à lâcher du lest pour rétablir les relations sociales ébranlées par la crise : sous la pression des grèves, le suffrage universel, toutefois limité par la pluralité des voix aux censitaires, capacitaires et pères de familles

3. X. MABILLE, Histoire politique de la Belgique, $2^{\mathrm{e}}$ édition, Bruxelles, CRISP, 1992 ; B. CHLEPNER, Cent Ans d'histoire sociale en Belgique, $2^{2}$ édition, Éditions de l'ULB, Bruxelles, 1972.

4. J. NEUVILLE, La Condition ouvrière au XIXe siecle, tome I : L'Ouvrier objet, et tome $2: L '$ 'Ouvrier suspect, Bruxelles, éditions Vie ouvrière, 1977.

5. P. DELFOSSE, «Répression, ordre social et développement de la société belge (1848-1914)», in Généalogie de la défense sociale en Belgique (I880-1914), Travaux du séminaire M. FOUCAULT, textes recueillis par F. TULKENS, Bruxelles, éditions Story-Scientia, 1988, pp.211 et ss.

6. M.-S. DUPONT-BOUCHAT, «Stratégies de maintien de l'ordre en Belgique et en France au XIX ${ }^{\mathrm{e}}$ siècle : la doctrine de la défense sociale », in Historische Soziologie der Rechtswissenschaft, sous la direction d'Erk Volkmar Heyen, Frankfurt am Main, 1986, pp. 79-106.

7. M. LIEBMAN, Les Socialistes belges, 1885-1914, Histoire du mouvement ouvrier en Belgique, t. 3, Bruxelles, Éd.Vie Ouvrière, 1979. 
nombreuses, est voté en 1893. Vingt-huit députés socialistes entrent à la Chambre en 1894. Sous leur influence, les premières lois sociales sont votées, mais il faudra encore vaincre bien des résistances avant de voir consacrer dans la législation la protection de l'enfance (1912) et la scolarité obligatoire (1914).

La doctrine de la défense sociale élaborée dès 1886 par Adolphe Prins (Criminalité et répression, 1886) est adoptée sur le plan social avant d'être définitivement formulée sur le plan juridique (A. Prins, La Défense sociale et les transformations du droit pénal, 1910) ${ }^{8}$. La mise en auvre d'une politique de protection des faibles (ouvriers, femmes, enfants) s'amorce dès la fin des années 1880 : réglementation du travail des femmes et des enfants (1889), logements sociaux, règlements d'atelier, réparation des accidents du travail... et sur le plan pénal : loi sur la condamnation et la liberation conditionnelles (1888), réorganisation des institutions pénitentiaires pour enfants (1890).

En même temps se développe une politique de répression accrue contre les «meneurs», les récidivistes, les criminels endurcis, les alcooliques et les vagabonds: loi de 1891 sur le vagabondage, aggravation des sanctions prévues par le code pénal à l'égard des meneurs, des perturbateurs du repos public, y compris les leaders socialistes (1892), régime spécial pour les récidivistes (1893), réorganisation des forces de maintien de l'ordre ${ }^{9} .$.

Si l'ère Lejeune aboutit à sortir l'enfance du pénal et de la prison, et apparaît généralement dans l'historiographie comme une période de "progrès ${ }^{10} "$, il ne faut pas se leurrer sur les objectifs d'une stratégie de défense sociale qui - comme son nom l'indique - vise d'abord à défendre l'ordre social menacé et à garantir cet ordre contre les menées subversives des agitateurs politiques et de la criminalité galopante des périodes de crise ${ }^{11}$.

Au-delà des ruptures et des transformations des politiques pénales à l'égard des jeunes délinquants, il existe néanmoins une continuité. Continuité de la politique de prévention, déjà présente chez Ducpétiaux, mais généralisée à présent à l'ensemble des «enfants en danger " qu'il faut protéger contre la mauvaise influence du milieu familial mais aussi de la prison. Il s'agit donc de trouver une alternative à l'éducation pénitentiaire qui est loin de remplir le rôle que lui assignait Ducpétiaux, notamment celui de prévenir la récidive. L'échec des pratiques carcérales à l'égard des

8. F. TULKENS, «Adolphe Prins et la défense sociale", in Généalogie de la défense en Belgique, op. cit., pp. 17 et ss.

9. P. DELFOSSE, « Répression, ordre social », op. cit., pp. 211-230.

10. Cf. les critiques émises à l'égard des hagiographes de Lejeune : H. JASPAR, «Jules Lejeune et son cuvre de législation criminelle», Revue de droir pénal, 1911, pp. 257-269; L. DUPONT, "Jules Lejeune et la défense sociale », in Généalogie de la défense sociale, op. cit., pp. 77-88.

11. M.-S. DUPONT-BOUCHAT, «Stratégies de maintien de l'ordre », op. cit. 
mineurs délinquants est analysé selon deux grilles de lecture appliquées à deux types de populations différentes : les « jeunes en danger », c'est-à-dire l'immense majorité des enfants des classes défavorisées, confrontées à la misère, à l'alcoolisme et menacées de dégénérescence, et d'autre part celle plus réduite des récidivistes, des incorrigibles, sur lesquels, dit Lejeune «les causes de dépravation ont achevé leur œuvre et qui devront être isolés comme de malheureux pestiférés ${ }^{12}$ ".

Continuité, donc, dans la répression, mais limitée cette fois aux seules natures rebelles pour lesquelles on n'espère même plus le redressement. Tandis que les premiers bénéficieront des mesures de protection et d'éducation dans les Écoles de bienfaisance, désormais soustraites à la tutelle de l'administration pénitentiaire pour être confiées à l'administration de la bienfaisance, les seconds seront rejetés dans les quartiers spéciaux pour incorrigibles ou vers les prisons d'adultes comme «irrécupérables ${ }^{13}$.

Parallèlement à l'œuvre d'éducation entreprise dans les Écoles de bienfaisance, les comités de patronage mis en place sous le contrôle du ministre de la Justice doivent relayer et poursuivre la politique de réinsertion des jeunes détenus libérés en assurant leur bien-être à la fois matériel et moral. C'est à eux qu'il incombera de choisir de "bonnes familles» pour le placement, en veillant soigneusement aux garanties morales qu'elles présentent pour éviter aux enfants de retomber dans les tares de leur milieu d'origine. Voilà tout au moins la théorie.

Mais on verra que, dans la pratique, le placement reste minoritaire et que l'enfant libéré n'a souvent d'autre ressource que de rentrer dans sa propre famille. C'est la raison pour laquelle le projet de loi sur la protection de l'enfance prévoit un contrôle des familles populaires, explicitement visées lors des débats sur la déchéance de la puissance paternelle.

En fonction des besoins sociaux et des idéologies dominantes propres à chaque époque, les institutions pénitentiaires pour mineurs ont donc évolué de la prison vers l'école de bienfaisance. Ce sont les étapes de ce cheminement qui s'étale sur plus d'un siècle que l'on retracera ici à partir de l'histoire de trois institutions: le pénitencier de Saint-Hubert, la première prison pour enfants créée en Belgique par Ducpétiaux en $1840^{14}$, la succur-

12. J. LEJEUNE, A.R. du 7 juillet 1890, Rapport au Roi.

13. Création du quartier spécial pour incorrigibles à la prison de Gand (A.R. 1887)

14. M.-S. DUPONT-BOUCHAT, «Le Pénitencier de Saint-Hubert, I. La Maison pénitentiaire pour jeunes délinquants, 1840-1867 ", in Saint-Hubert d'Ardenne, Cahiers d' histoire, t. V, 1981, pp. 161-182 ; «Il. De la maison pénitentiaire à l'école de bienfaisance (1867-1890)», in Saint-Hubert d'Ardenne, Cahiers d'histoire, t. VI, 1982, pp. 139-186; "IIl. L'école de bienfaisance (1890-1914)", Saint-Hubert d'Ardenne, Cahiers d'histoire, t. Vll, 1984, pp. 165-196 et « Saint-Hubert, pénitencier modèle au XIXe siècle ", in Saint-Hubert d'Ardenne, Cahiers d'histoire, t. VIII, 1991, pp. 143160 . 
sale du pénitencier de Saint-Hubert à Namur $(1871-1896)^{15}$ et le pénitencier pour filles installé à Liège (1848), puis à Namur $(1864)^{16}$.

\section{Saint-Hubert, pénitencier modèle au XIX'e siècle (1840-1914)}

L'histoire institutionnelle de la maison de Saint-Hubert est rythmée par les réformes successives qui introduisent à chaque fois un changement de dénomination du pénitencier. Ces différentes appellations témoignent à la fois du souci d'adapter les théories aux pratiques, compte tenu des diverses catégories de population qui y sont successivement détenues, en même temps qu'elles révèlent l'évolution de l'idéologie et de la politique pénitentiaires qui se transforment sous l'impulsion des besoins de la pratique.

La logique des moyens, combiné à celle des besoins, donne naissance à de nouvelles formulations «théoriques» de politique pénitentiaire à l'égard des mineurs délinquants qui se traduisent par de nouvelles dénominations. Celles-ci révèlent aussi les transformations du regard porté sur la délinquance juvénile, le passage d'une conception de l'enfance coupable à celle de l'enfance en danger. Ces changements de dénominations serviront donc de fil conducteur à l'analyse des transformations institutionnelles.

D'abord conçue comme une prison, la première prison pour enfants en Belgique, la maison s'appelle à l'origine, de 1840 à 1867, «Maison pénitentiaire des jeunes délinquants ».

L'accent est mis sur l'aspect répressif, l'enfance coupable, la prison ${ }^{17}$.

Suite aux réformes introduites par le code pénal de 1867 , et conformément aux vœux de Ducpétiaux qui rêvait de séparer les enfants condamnés de ceux qui avaient été acquittés et mis à la disposition du gouvernement, la maison pénitentiaire, qui accueillait encore les deux catégories, est rebaptisée en 1867 : Maison pénitentiaire er de réforme (1867-1881).

Fondée sur les critères du code pénal qui distingue les enfants «coupables » - parce que capables de discemement, donc responsables - des enfants non coupables, mais qui doivent être internés pour être moralisés et éduqués, «réformés», la double appellation de «pénitentiaire » est appliquée aux condamnés, coupables qu'il faut punir, tandis que celle de «maison de réforme " vaut pour les acquittés qu'il faut éduquer.

15. V. STRIMELLE, La Succursale du pénitencier de Saint-Hubert à Namur (1871-1896), mémoire de licence en histoire, UCL, Louvain-la-Neuve, 1985 (inédit).

16. F. ALEXANDRE, Le Pénitencier des jeunes délinquantes à Namur (18641890), mémoire de licence en histoire, UCL, Louvain-la-Neuve, 1987 (inédit).

17. M.-S. DUPONT-BOUCHAT, «Le Pénitencier de Saint-Hubert, I. La Maison pénitentíaire $»$, op. cit. 
À partir de 1881 , l'échec de la moralisation devient patent pour une catégorie d'enfants reconnus et déclarés «incorrigibles». Un quartier spécial est créé pour eux à la prison de Gand. Ce qui entraîne une nouvelle répartition des enfants entre les institutions. La maison de Saint-Hubert, qui devrait désormais n'être plus affectée qu'aux seuls acquittés, s'appellera «maison spéciale de réforme» jusqu'en 1890.

L'émergence, dans les années 1880 , des nouvelles théories criminologiques qui centrent leur analyse sur le danger de la récidive et les moyens à mettre en œuvre pour lutter préventivement contre elle, focalisent le débat sur l'enfance. L'utilisation des statistiques criminelles semblerait démontrer que les récidivistes se recrutent parmi ceux qui, enfants déjà, ont connu le pénitencier. L'enfant délinquant devient la cible de la prévention. En même temps, le critère pénal de discernement est abandonné au profit d'un critère social : l'enfant en danger remplace l'enfant coupable. Mais un nouveau critère psychologique apparaît et se traduit par la constitution d'une nouvelle catégorie : l'enfant incorrigible. La réforme introduite par J. Lejeune en 1890 aboutit donc à balayer toutes les anciennes catégories et dénominations, pour rebaptiser toutes les institutions d'un nom unique : les Écoles de bienfaisance de l'État.

Dénomination particulièrement significative parce que la priorité est accordée à l'éducation (école) et qu'il s'agit d'une œuvre de bienfaisance à l'égard des enfants les plus démunis. Premier pas pour sortir les enfants du pénal, « décriminaliser » la délinquance juvénile, en la considérant comme une maladie sociale, non plus imputable à la responsabilité d'un enfant "coupable", mais à une pathologie sociale, de la famille et du milieu, qui met l'enfant en danger.

La direction des écoles de bienfaisance est retirée à l'administration pénitentiaire pour être confiée à celle de la bienfaisance. Néanmoins, on conserve les quartiers spéciaux pour incorrigibles et on les multiplie en créant des quartiers spéciaux de discipline dans les écoles de bienfaisance, pour les malheureux sur lesquels, dit Lejeune, "la dépration a achevé son œuvre», en clair : des irrécupérables. Le débat qui s'élève sur cette question au Congrès pénitentiaire international de Saint-Pétersbourg, en $1890^{18}$, voit s'affronter deux conceptions : enfants « incorrigibles » ou

18. Congrès pénitentiaire international de Saint-Pétersbourg, 1890: Débat entre Prins, Stevens et Conception Arenal en réponse à la sixième question : «Peut-on admettre que certains criminels et délinquants soient considérés comme incorrigibles, et, dans l'affirmative, quels moyens pourraient être employés pour potéger la société contre cette catégorie de condamnés? S'agit-il "d'incorrigibles" ou de "non corrigés" »? Sur onze rapports présentés sur cette question, cinq rapporteurs penchent pour l'incorrigibilité, et six la rejettent (Actes du Congrès pénitentiaire international de Saint-Pétersbourg, 1890 , t. I. Saint-Pétersbourg, 1892, séance du 18 juin 1890, pp. 329-357, et conclusions, pp. 621-625). 
enfants «incorrigés"? C'est tout le problème de l'échec du système pénitentiaire qui est ainsi posé, et Lejeune choisit à partir de là de trier les enfants selon leur degré de «perversité », de dangerosité, et non plus de culpabilité. Il ne s'agit plus tellement de punir, mais d'éduquer ceux qui sont encore éducables, d'isoler «comme de malheureux pestiférés " ceux qui sont irrécupérables.

Tout ce débat et le changement de dénomination qui s'ensuit est extrêmement révélateur non seulement de l'évolution des théories pénales et criminologiques, mais aussi du climat de l'époque : montée des mouvements sociaux, danger social, nouvelles stratégies de maintien de l'ordre : protéger les «bons", poursuivre les «mauvais", les meneurs; c'est toute l'idéologie de la défense sociale. Et aussi l'héritage de la philanthropie désormais prise en charge par l'État. «École de bienfaisance de l'État» traduit la conversion de l'État-gendarme en État paternaliste, philanthrope et bienveillant, enfin plus conscient de sa mission sociale à l'égard des plus faibles et des plus démunis, en l'occurrence les enfants, sur lesquels il s'agit de veiller avec bienveillance, bienfaisance, pour éviter qu'ils ne retombent dans l'armée de la récidive et qu'ils ne viennent encore grossir les rangs des criminels «d'habitude».

Le vocabulaire de l'époque témoigne parfaitement des préoccupations et des enjeux politiques et sociaux, d'une nouvelle conception du maintien de l'ordre.

Saint-Hubert, comme toutes les autres institutions pour mineurs, s'appellera «école de bienfaisance » jusqu'en 1921. À cette date, le terme de «bienfaisance », tombé en désuétude, sera remplacé par celui d' "éducation", et le terme d'《école " par celui d' «établissement» : Établissement d'éducation de l'État.

L'école a définitivement triomphé de la prison, l'éducation de la répression. L'État qui a toujours géré ces institutions, quel que soit leur nom, en revendique désormais la paternité. Le débat sur la déchéance de la puissance paternelle (1889-1912) posait la même question : qui est propriétaire de l'enfant? Le père ou l'État? Les catholiques accusaient les socialistes de vouloir «voler» les enfants à leur propriétaire légitime, le père, en subsituant l'État à celui-ci pour décréter ce qui était le bien de l'enfant (par exemple aller à l'usine ou à l'école). D'où les interminables débats sur la réglementation du travail des enfants, mais surtout sur l'obligation scolaire (loi de 1914). Enfin, on se mit d'accord: les enfants pauvres, ceux des familles ouvrières, dont les pères seraient déficients, et qui seraient, de ce fait, «en danger», seront placés sous l'autorité tutélaire d'un nouveau père : l'État.

Si ces changements de dénominations traduisent les transformations de l'idéologie et de la politique pénales, ils ne coïncident qu'imparfaitement 
avec des modifications profondes de la pratique d'enfermement, ou même des priorités de traitement. L'institution change de nom, mais à l'intérieur rien n'est changé, si ce n'est, parfois, la population, triée selon de nouveaux critères.

Il ne faut donc pas se laisser abuser par ces étiquettes, plus révélatrices d'intentions que de réalisations concrètes.

\section{1) La maison pénitentiaire pour jeunes délinquants $(1840-1867)^{19}$}

La création du pénitencier de Saint-Hubert est l'œuvre de Ducpétiaux ${ }^{20}$. On ne développera pas ici l'histoire des avatars et des difficultés rencontrées par le fondateur pour imposer une idée, un modèle qui représentait la synthèse de toutes les expériences menées à l'étranger, aux États-Unis, en Angleterre comme en France ou en Allemagne ${ }^{2 !}$.

Malgré tous ses efforts, Ducpétiaux n'avait pu faire triompher ses vues : il contestait le choix du site, l'ancienne abbaye de Saint-Hubert, qui impliquait l'adaptation de bâtiments qui n'avaient pas été conçus pour l'usage qu'il voulait en faire, l'éloignement par rapport à Bruxelles d'un pénitencier «central " qui échappait au regard direct de l'administration pénitentiaire, et enfin le caractère partiel de la mesure qui l'obligeait dans un premier temps à se contenter d'une seule institution alors qu'il en aurait fallu trois pour pouvoir séparer les condamnés des acquittés 22 .

Le gouvernement peu soucieux d'investir une fortune dans la construction de trois nouvelles institutions, imposa finalement Saint-Hubert, à la fois pour d'évidentes raisons d'économie, mais aussi pour des motifs de politique locale. Lors de l'occupation française, Saint-Hubert avait été le chef-lieu temporaire de l'administration du département des Forêts, avant de devenir une sous-préfecture du département de Sambre-et-Meuse. Un tribunal de première instance y avait été installé. Après la Révolution belge de 1830 , elle perdit son tribunal qui fut transféré à Neufchâteau, en même temps qu'elle devait abandonner tout espoir de devenir le chef-lieu de la

19. Pour toute cette partie, et pour plus de détails, on se reportera à M.-S. DUPONT-BOUCHAT, «Le pénitencier de Saint-Hubert. I. La Maison pénitentiaire pour jeunes délinquants ", op. cit.

20. Sur Ducpétiaux, cf. notamment M.-S. DUPONT-BOUCHAT, « Ducpétiaux ou le rêve cellulaire», in Déviance et Société, Genève, 1988, vol. 12, no 1, pp. 1-27; T. PETERS, «Édouard Ducpétiaux», in C. FIJNAUT, Gestalten uit het verleden, Kluwer Belgie, 1993, pp. 33-49.

21. M.-S. DUPONT-BOUCHAT, «Saint-Hubert, pénitencier modèle au XIXe siècle »,op. cit., pp. 144-150: « Saint-Hubert ou la difficile synthèse des modèles belge, américain et français ».

22. É. DUCPETIAUX, Mémoire pour l'établissement d'un pénitencier central à Saint-Hubert. Bruxelles, 1840, pp. 50-54. 
province de Luxembourg dont le siège fut fixé à Arlon en 1839. Le gouvernement provincial trouva une occasion de dédommager la ville en votant, à l'unanimité, le 10 juillet 1840, l'abandon gratuit des bâtiments de l'ancienne abbaye à l'État, à condition d'y établir le pénitencier ${ }^{23}$.

C'était là une manière de se débarasser sur l'État de la charge que représentait l'entretien de ces bâtiments. Après deux ans de tergiversations, suite aux contestations de Ducpétiaux, le gouvernement accepta la cession des locaux de l'abbaye en s'engageant à libérer une somme de 300000 francs pour l'aménagement et l'appropriation des bâtiments à leur nouvelle destination ${ }^{24}$.

Dès lors, la machine administrative est lancée : le 8 octobre 1843 est créé un comité d'inspection et de surveillance de la maison pénitentiaire. $\mathrm{Vu}$ la distance qui sépare Saint-Hubert de l'administration centrale, une dérogation aux règles en vigueur est autorisée : ce comité sera composé de six membres tous recrutés sur place. Le président de cette commission est Schmidt, doyen-curé de Saint-Hubert, les membres sont des notables locaux, parmi lesquels figurent le député Zoude, le juge de paix Lambin, l'inspecteur des contributions Sohier, qui est également députê provincial, tous habitants de Saint-Hubert. Le bourgmestre et le procureur du Roi sont membres de droit de ce comité ${ }^{25}$.

Le règlement d'ordre du comité est promulgué le 15 mai $1844^{26}$. I] définit le fonctionnement du comité de surveillance et les tâches qu'il aura à accomplir : c'est lui en fait qui sert de courroie de transmission entre le directeur et le ministre de la Justice. Sa mission concerne tous les aspects du régime intérieur de la maison depuis l'entretien des bâtiments et l'approvisionnement, jusqu'au travail, l'instruction, la réforme des détenus. Il fait au ministre toutes les propositions qu'il juge convenables dans I'intérêt du pénitencier et peut, en cas d'urgence, se substituer à celui-ci pour donner des ordres. Il se réunit au moins une fois tous les quinze jours et charge un de ses membres d'inspecter, au moins une fois par semaine, le pénitencier. Il vérifie les dépenses chaque mois et établit chaque année un projet de budget qu'il soumet au ministre. Enfin, il rédige un rapport annuel sur la situation de la maison qui doit reprendre au début de chaque année le tableau général de la population, les dépenses, l'inventaire du mobilier et

23. L. HANNECART, «Saint-Hubert», in Communes de Belgique. Dictionnaire d'histoire et de géographie administrative, t. II, p. 1310.

24. Recueil des Circulaires du Ministère de la Justice (R.C.M.J.)., 3e série, 18401845 : Cession à l'État de l'ancienne abbaye de Saint-Hubert, rapport au Roi sur la décision du Conseil provincial du 19 juillet 1840, Bruxelles, 14 janvier 1842, signé de Van Volxem, ministre de la Justice., et AR du 17 janvier 1842.

25. RCMJ, 8 octobre 1843 , et règlement du 15 mai 1844.

26. R.C.M.J., 14 articles, 15 mai 1844. 
des effets d'habillement, un êtat des ateliers, un tableau de la situation sanitaire, un compte moral reprenant toutes les punitions et récompenses, ainsi que les progrès effectués par les détenus. Ce rapport général sera lui même fondé sur tous les rapports particuliers établis par les frères-surveillants, l'aumônier, le médecin, le directeur.

Une organisation strictement hiérarchisée est ainsi mise en place : elle part au sommet du ministre de la Justice, relayé à l'échelle locale par le comité de surveillance qui doit contrôler le directeur; celui-ci chapeaute toute l'administration interne répartie selon trois secteurs : le supérieur des frères, responsable de la surveillance, de l'école et des ateliers, l'aumônier et le médecin. Toute une cascade de rapports quotidiens, hebdomadaires, mensuels, annuels est prévue pour faire circuler d'un échelon à l'autre toutes les informations qui concernent chaque secteur de la vie quotidienne. Rien ne doit être laissé dans l'ombre : tout doit être minutieusement enregistré, comptabilisé, vérifié, contrôlé, qu'il s'agisse du fonctionnement matériel (bâtiments, nourriture, habillement, mobilier, ateliers, livres de la bibliothèque), de la santé physique (état sanitaire, alimentation, maladies, décès), mais surtout de la santé morale (discipline, récompenses, punitions, progrès individuels). Le tout sera strictement comptabilisé, statistiques à l'appui, au grand livre de la «comptabilité morale».

Sources fabuleuses pour l'historien, à première vue du moins, car, à l'usage, il se révèle que cette obsession administrative ne produit pas dans la pratique tous les documents escomptés et, surtout, que lorsqu'ils existent, ils n'alignent qu'une foule de petits détails secondaires, l'essentiel n'étant jamais dit.

C'est donc à partir de ces petites touches ponctuelles que l'historien doit reconstruire la logique qui préside aux choix de la gestion quotidienne, parfois fort éloignés des principes et des intentions définis par les circulaires ministérielles. Celles-ci ne cessent de pleuvoir sur les responsables de l'établissement pour organiser de loin, abstraitement, une gestion locale qui doit fatalement composer avec des impératifs beaucoup plus terre à terre, ceux qui s'expriment justement dans les résidus des archives de la pratique. Recettes pour prévenir les coliques, ou mérites comparés du pain de seigle ou de son, entretien des parquets rongés par l'urine des enfants : voilà les vrais problèmes de la gestion quotidienne qui apparaissent au fil des dépouillements.

L'écart entre les objectifs élevés du promoteur et de l'administration pénitentaire et les préoccupations des gestionnaires locaux se mesure à travers les archives de la pratique. Surveiller et punir, mais surtout éduquer et moraliser pour les premiers ; loger, nourrir, soigner, occuper, réprimer et sévir pour les seconds... 
Le 20 mai 1844, un arrêté royal fixe la destination du pénitencier. Y seront enfermés :

$1^{\circ}$ les enfants mâles âgés de moins de seize ans condamnés à un emprisonnement de six mois et plus ;

$2^{\circ}$ les enfants acquittés comme ayant agi sans discernement et mis à la disposition du gouvernement, conformément à l'article 66 du code pénal de 1810 .

$3^{\circ}$ Le ministre de la Justice pourra en outre y faire transférer les condamnés âgés de plus de seize ans, mais de moins de vingt et un ans, âge à partir duquel les condamnés qui n'auront pas achevé de purger leur peine seront renvoyes dans les prisons criminelles ou correctionnelles ${ }^{27}$.

L'affectation du pénitencier à ces deux catégories d'enfants, condamnés et acquittés, allait à l'encontre des projets de Ducpétiaux qui aurait voulu les séparer, de peur que les premiers ne contaminent les seconds, réputés moins mauvais, puisque non-coupables. En outre, comme Saint-Hubert est le premier établissement à être ouvert en Belgique, on y transfère également des petits vagabonds et mendiants jusque-là enfermés avec les adultes dans les dépôts de mendicité.

La politique pénitentiaire du fondateur qui réclamait trois types d'institutions distinctes est ainsi bafouée dès l'origine.

Le $1^{\text {er }}$ juin 1844 , le pénitencier ouvre ses portes au premier contingent de jeunes détenus : ils sont soixante-six venant du quartier spécial de SaintBernard ou des maisons de sûreté ou d'arrêt où ils étaient retenus en attendant l'ouverture du nouvel établissement. La population s'accroît rapidement : dès le $1^{\text {er }}$ janvier 1845 , elle compte 133 détenus, dont 96 condamnés et 37 acquittés $^{28}$. Entre 1844 et 1852, il entrera 1234 enfants à Saint-Hubert ${ }^{29}$.

Les années de crise et de misère que traverse la Belgique dans les années 1845-1848 voient augmenter de manière impressionnante le nombre des enfants écroués : des malheureux vont jusqu'à demander asile dans les prisons pour échapper au froid, à ja faim, à la mort. Le nombre des jeunes détenus enfermés dans les prisons et les dépôts de mendicité passe de 4398 en 1845 à 8800 en 1846 et 13049 en 1847 .

Sur un total de 26247 pour ces trois années, 17813 se trouvent dans les prisons d'adultes et 8434 dans les dépôts de mendicité ${ }^{30}$.

27. R.C.M.J., A.R. 20 mai 1844, Destination du pénitencier de Saint-Hubert.

28. É. DUCPETIAUX, Notice statistique sur la maison des jeunes délinquants de Suint-Hubert, Bruxelles, 1852, p. 9.

29. Ibid.

30. J. NEUVILLE, La Condition ouvrière au XIX' siècle, t. II : l'ouvrier suspect. Bruxelles, 1977, p. 67. 
D'après Ducpétiaux, cependant, l'augmentation du nombre d'internements tient également à une nouvelle attitude des tribunaux vis-à-vis des enfants délinquants : avant la création de Saint-Hubert, ils hésitaient à les condamner de peur de les envoyer en prison avec les adultes, par crainte de les contaminer ; désormais, ils se montrent plus sévères allant jusqu'à allonger la durée de l'enfermement dans «l'intérêt » des enfants coupables ${ }^{31}$.

La conjonction de ces deux facteurs aboutit très vite à l'engorgement de Saint-Hubert. Dès les premières années de son existence, la maison ne peut plus faire face aux flots successifs d'enfants : 258 entrées en 1846, 294 dans les premiers mois de 1847 . Au $1^{\mathrm{er}}$ septembre de cette année, la population de Saint-Hubert s'élève à 458 détenus, chiffre «excessif » dépassant la capacité d'accueil du pénitencier (conçu pour 350 à 400 enfants au maximum). Jusqu'en mars 1848, l'administration pénitentiaire est contrainte de suspendre l'envoi des jeunes condamnés à Saint-Hubert. Ceux-ci sont à nouveau dirigés vers les prisons pour adultes, au grand dam de Ducpétiaux. Au $1^{\text {er }}$ janvier 1849, il reste toujours 419 enfants dans la maison et c'est beaucoup trop : 49 sont malades, 17 sont morts en 1847 et 10 en $1848^{32}$.

C'est dans ce contexte que sera votée la loi du 3 avril 1848 sur les dépôts de mendicité et les écoles de réforme. Les premiers seront exclusivement réservés aux adultes, tandis que l'on créera pour les jeunes indigents de moins de dix-huit ans des "écoles de réforme». Deux établissements sont prévus, un pour les garçons à Ruiselede et un pour les filles à Beernem. Ils sont destinés aux enfants qui y entrent «volontairement» (c'est-à-dire ceux qui sont placés par les administrations communales qui financent leur entretien) ou qui auront été condamnés du chef de vagabondage et de mendicité. Les enfants y seront employés aux travaux des champs. La durée de l'internement sera de six mois au moins pour la première fois, d'un an au moins en cas de récidive ${ }^{33}$.

L'ouverture de l'école de réforme de Ruiselede en 1849 réalise un des vœux de Ducpétiaux: la séparation entre les enfants malheureux, vagabonds ou mendiants, et les enfants coupables, condamnés par les tribunaux, avec lesquels ils étaient jusque-là confondus au pénitencier.

31. É. DUCPETIAUX, Notice statistique, p. 9.

32. lbid., tableau statistique, pp. 10-11.

33. Pasinomie, loi du 3 avril 1848, art. 5 et 6 ; A.R. du 8 mars 1849 qui institue l'école de réforme de Ruiselede pour les garçons, et A.R. du 28 mars 1852 qui institue l'école de réforme pour les filles à Beernem. Sur l'histoire de cette institution, cf. A. MORTIER, Un siècle d'histoire de l'établissement d'éducation de l'État à Ruiselede. Office de la protection de l'enfance, s. d. 
En mai 1849, 65 jeunes indigents quittent Saint-Hubert pour Ruiselede "débarrassant le pénitencier d'un élément qui devait lui rester étranger ${ }^{34}$ ", dit Ducpétiaux.

Mais cette mesure ne suffit pas à elle seule à désencombrer la maison ni à réaliser la séparation entre acquittés et condamnés qui n'est toujours pas résolue. L'exposé des motifs de l'arrêté royal du 29 septembre 1848 autorisant la mise en apprentissage des jeunes acquittés chez des cultivateurs ou des artisans, ou encore dans des établissements privés de charité, déclare explicitement: "L'établissement de Saint-Hubert ne pouvant établir la séparation entre acquittés et condamnés, et comme il n'existe aucune autre maison destinée aux premiers, il vaut mieux placer ceux-ci en apprentissage.»

En 1855, la mesure sera encore étendue par l'autorisation d'engager des jeunes délinquants comme mousses à bord des navires de la marine marchande ou de les employer dans les mines, "tant dans l'intérêt des enfants (sic) que dans celui des exploitants ${ }^{35} \gg$ !

La volonté de réserver désormais Saint-Hubert aux seuls condamnés est réaffirmée dans les exposés des motifs justifiant ces décisions; elle est encore confirmée en 1850 par l'ouverture du pénitencier à une catégorie jusque-là fort peu représentée, les condamnés de seize à dix-huit ans.

Mais en 1855, la politique va changer sous la double contrainte de la pression démographique et de la logique de séparation : une succursale de Saint-Hubert est ouverte à la prison militaire d'Alost pour les condamnés, et le pénitencier ne devrait plus être réservé qu'aux seuls acquittés ${ }^{36}$. L'exposé des motifs à l'appui de cette mesure fait une fois de plus état de la surpopulation du pénitencier: "Depuis plusieurs années, la maison pénitentiaire de Saint-Hubert se trouve dans un état d'encombrement quasi permanent (...) Pour remédier à cet état de choses, il paraît convenable de créer à la maison militaire d'Alost un quartier entièrement séparé qui puisse servir, du moins provisoirement, de succursale à la maison de SaintHubert. »

La mesure sera en effet toute "provisoire", puisque cette annexe est fermée quatre ans plus tard ${ }^{37}$.

Les premières années d'existence de la maison pénitentiaire de SaintHubert sont essentiellement marquées par la nécessité de répondre à l'augmentation constante de la population, en liaison avec la conjoncture

34. É. DUCPETIAUX, Notice statistique, p. 10.

35. R.C.M.J., A.R. du 29 septembre 1848 et du 7 décembre 1855.

36. R.C.M.J., A.R. du 8 mai 1855 ; circulaires d'application des 8 et 20 août 1855 .

37. R.C.M.J., A.R. du 9 juillet 1859. 
économique et sociale, mais aussi avec le changement d'attitude des juges face aux jeunes délinquants.

La logique des besoins s'impose prioritairement contre les idéaux du fondateur sans que ceux-ci soient oubliés pour autant. Toute l'argumentation qui soutient les décisions, parfois contradictoires, de l'administration pénitentiaire fait droit aux deux objectifs: soulager le pénitencier de la pression démographique, jusqu'en envoyant - «pour leur plus grand bien" - les enfants dans les mines, et en même temps séparer les condamnés des acquittés, en prévoyant, selon les cas, d'affecter la maison pénitentiaire aux premiers ou aux seconds.

Le premier objectif de Ducpétiaux était de séparer les enfants des adultes. Il n'est lui aussi que partiellement rempli : certes, il existe dès 1844 une prison pour les enfants et dès 1849 et 1852 deux écoles de réforme pour les jeunes indigents. Mais bon nombre de jeunes délinquants sont toujours enfermés dans les prisons pour adultes et la tentative de les séparer par la création de la succursale d'Alost est vouée à l'échec, même s'il est prévu d'isoler le quartier des jeunes de la prison militaire.

L'incompatibilité entre le rêve de Ducpétiaux et les besoins sociaux vont amener l'administration à adapter la dénomination du pénitencier à la réalité de sa double destination.

\section{2) La maison pénitentiaire et de réforme (1867-1881)}

L'Arrêté royal du 16 décembre 1867 qui modifie la dénomination de la maison pénitentiaire de Saint-Hubert coïncide avec l'adoption du nouveau code pénal qui prévoit que les enfants acquittés, s'ils ne sont pas remis à leurs parents, doivent être envoyés dans des établissements spéciaux de réforme pour y être éduqués.

Le pénitencier de Saint-Hubert s'appellera désormais Maison pénitentiaire et de réforme, conformément aux deux types de population qu'il continue à accueillir: les «condamnés » qu'il faut punir et les «acquittés» qu'il faut « réformer».

La double dénomination témoigne toujours du souci d'adapter les «théories» aux pratiques, puisque la séparation entre les deux catégories d'enfants demeure impossible en l'absence d'une autre institution spécialisée. La fermeture de la succursale d'Alost qui aurait dû être réservée aux seuls condamnés n'a pas permis de réaliser le væu de Ducpétiaux.

Conjointement à cette volonté de respecter les objectifs pénitentiaires du réformateus, se pose encore de façon plus cruciale le problème de la surpopulation de Saint-Hubert.

La tentative de résoudre en même temps les deux problèmes par la création d'une succursale à Alost ayant échoué, l'administration péniten- 
tiaire envisage une nouvelle solution en profitant de la fermeture de la prison des femmes à Namur.

L'adoption, en 1870, de la loi sur le cellulaire qui généralisait l'enfermement séparé pour toutes les catégories de détenus, à l'exception des mineurs, et pour autant qu'il y ait des places disponibles dans les différentes prisons, eut pour conséquence d'entraîner la fermeture de la prison pour femmes créée à Namur par Ducpétiaux en 1837. Celles-ci seraient désormais renvoyées dans les prisons cellulaires où des quartiers distincts leur seraient réservés ${ }^{38}$. Ce départ libérait ainsi des places pour d'autres catégories de prisonniers. La population féminine de la prison de Namur, qui avait hébergé jusqu'à 526 détenues en 1855, n'avait cessé de décroître depuis 1864 où elle ne comprenait plus que 290 prisonnières. C'est à ce moment que l'on avait transféré à Namur le pénitencier pour filles, jusquelà situé à Liège ${ }^{39}$. En 1870 , le nombre de femmes détenues à Namur n'était plus que de $138^{40}$. Leur transfert vers les prisons cellulaires rendait possible la réaffectation des locaux aux jeunes délinquants excédentaires de Saint-Hubert. Ceux-ci allaient donc rejoindre à Namur, mais dans des quartiers séparés, les filles qui y avaient été transférées de Liège en 1848.

On assiste donc en 1871 à une sorte de chassé-croisé qui remplace une population pénitentiaire par une autre dans les bâtiments devenus disponibles à la suite du déménagement des femmes. Cela ne va pas simplifier la tâche de l'administration, ni celle du chercheur d'ailleurs, puisqu'on se trouve sans cesse confronté, suite à ces mutations successives, à la production de nouvelles archives, émanant d'une institution qui abrite en fait deux pénitenciers séparés mais réunis sous le même toit, avec une même direction, mais des personnels différents pour les garçons et pour les filles. On traitera dans le chapitre suivant de la succursale de Namur en distinguant les deux populations et l'on se bornera ici à tenter de mesurer l'impact de cette nouvelle création sur le pénitencier de Saint-Hubert.

Dans son Rapport au Roi à l'appui de l'Arrêté Royal du 27 avril 1871 qui crée à la prison de Namur une succursale de Saint-Hubert, le ministre de la Justice, Prosper Cornesse, justifie cette mesure par la nécessité de réduire la population de Saint-Hubert, toujours excédentaire, et de respecter le principe de la séparation entre condamnés et acquittés: les premiers devraient être transférés à Namur et internés dans les cellules rendues

38. S. LAURENT, La Première Prison pour femmes en Belgique. Namur, 1837. 1871 , pp. 46-47, loi du 4 mars 1870, arrêtés d'application des 27 avril et 16 mai 1871 .

39. Cf. ci-dessous la succursale de Namur.

40. S. LAURENT, op. cit., p. 79 : il faut noter la difference entre les chiffres fournis par les registres d'écrou (138) et par L'Exposé de la situation de la province de 1870-1871, qui indique 212, en globalisant les détenues adultes et les adolescentes enfermées à Namur. 
disponibles par le départ des femmes, tandis que Saint-Hubert devrait être réservé aux seuls acquittés ${ }^{41}$.

Si l'on tente de mesurer l'impact démographique de cette création sur la population du pénitencier de Saint-Hubert, il faut bien constater qu'il est pratiquement sans effet et, en tout cas, de courte durée. L'ouverture successives de nouveaux quartiers à Alost (en 1855), à Namur (en 1871) et plus tard à Gand (1881), puis pour les incorrigibles (1887), ne réduit pas de façon substancielle et encore moins définitive la surpopulation de SaintHubert, comme le montre le tableau ci-dessous.

Tableau 1

Mouvements de population au pénitencier de Sainf-Hubert suite à l'ouverture des diverses succursales

\begin{tabular}{|c|c|c|}
\hline Dates & Population & Remarques \\
\hline 31 décembre 1854 & 392 & \\
\hline 31 décembre 1855 & 375 & après l'ouverture d'Alost \\
\hline 31 décembre 1870 & 463 & \\
\hline 31 décembre 1871 & 374 & après l'ouverture de Namur \\
\hline 31 décembre 1872 & 400 & \\
\hline 31 décembre 1880 & 453 & \\
\hline 31 décembre 1881 & 442 & après l'ouverture de Gand \\
\hline 31 décembre 1882 & 483 & \\
\hline
\end{tabular}

Ces chiffres ne portent que sur la population présente au pénitencier à la date du 31 décembre, mais il est évident que le nombre d'enfants qui passent par Saint-Hubert en un an est voisin, sinon supérieur, à cinq cents. C'est d'ailleurs la norme, selon le directeur Stevens : «Cinq cents pensionnaires, c'est la population normale du pénitencier ${ }^{42}$.»

Les mesures qui visent à désengorger le pénitencier n'ont guère d'effet, puisque la population reste relativement constante et trop importante pour une capacité maximale d'accueil que Ducpétiaux situait autour de quatre cents.

Le second problème, celui de la séparation entre condamnés et acquittés, n'est pas davantage résolu. Pendant les quatre années d'occupation de la succursale d'Alost (1855-1859), le registre d'inscriptions de cette maison révèle la présence des deux catégories d'enfants, bien que, théoriquement, Alost aurait dû être réservé aux seuls condamnés ${ }^{43}$. En 1867 , Saint-Hubert

41. R.C.M.J., A.R. du 27 avril 1867.

42. Joumal des Tribunaux, $n^{\circ} 47$, col. 762 .

43. J. VAN GASSE, Les Érablissements pour jeunes délinquants en Belgique (1840-1912), Memoire de licence ULB, 1980-1981, inédit, p. 173 (chiffres tirés du registre d'inscriptions à la prison d'Alost, Archives de l'État à Beveren-Waas, Aalst gevangenis, $n^{\circ} 187$ ). 
accueille toujours les deux catégories d'enfants, comme en témoigne la double appellation «pénitentiaire et de réforme».

La circulaire ministérielle du 16 mai 1871 qui fait suite à la création de la succursale de Namur prévoit une nouvelle répartition des enfants selon dix catégories.

A posteriori, on peut considérer cet effort de classification pointue comme le point ultime de la politique préconisée par Ducpétiaux. Ses successeurs, fidèles à ses principes, s'efforcent de construire un chef-d'œuvre d'architecture institutionnelle théorique qui, on s'en doute, s'avérera totalement irréalisable dans la pratique. En essayant de combiner les différents critères d'âge, de provenance géographique (distinction entre milieu rural et urbain) et de statut pénal (condamnés ou acquittés), ils aboutissent à complexifier le classement de telle sorte qu'aucun directeur ne pourra s'y retrouver ni l'appliquer, d'autant que les dossiers d'enfants qui leur sont envoyés sont incomplets et ne permettent pas d'opérer ce tri. Les circulaires du 10 avril 1872 et du 29 juillet 1875 regrettent que les dossiers des jeunes détenus envoyés à Saint-Hubert soient absolument incomplets : y manquent les extraits de jugement, les actes de naissance, les dates auxquelles la détention ordonnée par jugement prend cours, les circonstances du délit, l'exposé des motifs de la condamnation, la gravité du préjudice causé, etc. ${ }^{44}$. L'administration pénitentiaire insiste auprès des procureurs généraux pour obtenir des renseignements complets afin de déterminer si le délinquant a exprimé des regrets face à son acte, ou si, au contraire, il est signalé comme "un malfaiteur endurci et indigne d'intérêt».

Ceci devant servir à compléter des dossiers de la comptabilité morale de chaque détenu, condamné à une peine d'un an et plus.

Même si les directeurs avaient voulu opérer le classement selon les principes de l'administration sur la base des renseignements foumis, c'eût encore été impossible, étant donné les capacités d'accueil des institutions existantes.

La circulaire du 16 mai 1871 représente un monument de la pensée administrative qui est loin d'être une exception, puisque ces circulaires sont fréquemment revues, complétées et corrigées.

Elle mérite néanmoins d'être présentée ici, à titre d'exemple et d'illustration des dernières tentatives de classement selon la logique pénale. On comprendra mieux, par la suite, la réforme de Lejeune qui abandonne tous ces critères pour créer un type d'institution unique : les «écoles de bienfaisance", où la répartition entre les différentes écoles se fera désormais essentiellement selon des critères d'âge.

44. R.C.M.J., circulaires des 10 avril 1872 et 29 juillet 1875. 
Enfin, la répartition prévue par la circulaire du 16 mai 1871 fait clairement apparaître l'âge limite jusqu'auquel joue le critère de discernement (16 ans) et le traitement différencié des différentes catégories de jeunes condamnés qui ont dépassé cet âge. Entre seize et dix-huit ans, les plus "pervertis", selon l'expression du temps, peuvent encore "bénéficier", s'ils sont condamnés à une peine longue (plus de six mois), à un enfermement «privilégié » dans la maison pénitentiaire et de réforme de Namur. Par contre, ceux de quatorze ans, comme ceux de seize ou de dix-huit, qui sont condamnés à des peines courtes, et donc considérés comme moins « coupables» ou moins pervers, sont dirigés vers les prisons pour adultes... Après dix-huit ans, les "criminels endurcis» sont expédiés à la prison centrale de Louvain et soumis au cellulaire.

Ce n'est plus là, tant s'en faut, respecter le vœu de Ducpétiaux, qui voulait à tout prix séparer les enfants des adultes.

Mais où finit l'enfance et où commence l'âge adulte? À quatorze ans, à seize ou à dix-huit? L'âge de la majorité pénale fera toujours l'objet de nombreux débats par la suite, notamment lors de l'élaboration de la loi de 1912, et plus tard lorsqu'il s'agira de revoir certaines dispositions de cette toi.

Néanmoins, on peut formuler ici deux autres types d'observations : tout d'abord que l'internement en maison de correction ou de réforme, considéré comme un privilège, n'est pas «rentable» pour les courtes peines, d'où la tendance à allonger la durée d'enfermement pour parfaire la moralisation et l'éducation, bref la «réforme » du jeune délinquant.

Deuxièment, que les seules institutions de Saint-Hubert et de Namur ne pouvaient évidemment suffire à absorber, pour une longue durée, tous les jeunes délinquants jugés en vertu des articles 72 et 76 du CP (acquittés) et des articles 73-74 (condamnés). Le recours à la prison s'avère incontournable aussi bien pour des motifs de sévérité accrue à l'égard de ceux qui sont considérés comme « responsables » que pour des motifs purement pratiques: la place disponible dans ces deux institutions spécialisées, perpétuellement encombrées.

Ceci amorce un autre débat et une ultime tentative du ministre de la Justice Jules Bara en 1881, qui se traduira, une fois encore par un nouveau changement de dénomination. Mais avant d'analyser cette troisième étape, voici le classement prévu en 1871 : 
Tableau 2

Tableau indicatif des règles sur le classement des jeunes délinquants, 16 mai 187 (Garçons)

\begin{tabular}{|c|c|c|}
\hline Âge & Catégorie & Établissement \\
\hline A. moins de 14 ans & $\begin{array}{l}1^{\text {re }} \text { catégorie : } \\
\text { acquittés et mis à la } \\
\text { disposition du } \\
\text { gouvernement (art. } 72 \text { et } 76 \\
\text { du CP) } \\
2^{\text {e }} \text { catégorie : } \\
\text { condamnés (art. } 73 \text { et } 74 \\
\text { CP) } \\
\text { a) plus de six mois } \\
3^{e} \text { catégorie : } \\
\text { condamnés (art. } 73 \text { et } 74 \text { ) } \\
\text { b) moins de six mois }\end{array}$ & $\begin{array}{l}\text { succursale de Namur } \\
\text { maison d'arrêt ou de sareté }\end{array}$ \\
\hline B. de 14 à 16 ans & $\begin{array}{l}4^{\mathbf{e}} \text { catégorie : } \\
\text { acquittés (art. } 72 \text { et } 76 \text { ) } \\
5^{\mathbf{e}} \text { catégorie : } \\
\text { condamnés à plus de } \\
\text { six mois } \\
6^{\mathbf{e}} \text { catégorie : } \\
\text { condamnés à moins de } \\
\text { six mois }\end{array}$ & $\begin{array}{l}\text { a) origine rurale : Saint-Hubert } \\
\text { b) origine urbaine : Namur } \\
\text { Namur } \\
\text { maison de sûreté ou d'arrêt }\end{array}$ \\
\hline C. de 16 à 18 ans & $\begin{array}{l}7^{\mathbf{e}} \text { catégorie : } \\
\text { condamnés aux travaux } \\
\text { forcés, à la réclusion ou à } \\
\text { un emprisonnement de plus } \\
\text { de six mois } \\
\mathbf{8}^{\mathbf{e}} \text { catégorie : } \\
\text { condamnés à moins de } \\
\text { six mois }\end{array}$ & $\begin{array}{l}\text { Namur } \\
\text { maison de sôreté ou d'arrêt }\end{array}$ \\
\hline D. de 18 à 21 ans & $\begin{array}{l}9 \text { catégorie : } \\
\text { condarnnés aux travaux } \\
\text { forcés, à la réclusion, à un } \\
\text { emprisonnement de plus de } \\
\text { deux ans } \\
10^{e} \text { catégorie : } \\
\text { condamnés à moins de } \\
\text { deux ans }\end{array}$ & maison de sûreté ou d'arrêt \\
\hline
\end{tabular}

\section{3) Saint-Hubert: Maison spéciale de Réforme (1881-1890)}

La troisième étape de l'histoire institutionnelle du pénitencier de SaintHubert marque l'aboutissement, en même temps que la faillite, de la logique de classement voulue par Ducpétiaux.

Pendant cette brève période de dix ans, où surgit partout en Europe et en Amérique du Nord une nouvelle réflexion sur l'enfance et la protection 
de l'enfance ${ }^{45}$ et où l'on élabore de nouveaux modèles institutionnels pour l'enfance délinquante, la Belgique, qui se situe à la pointe de ce mouvement, hésite encore entre le modèle pénal traditionnel et le nouveau modèle «protecteur». Les idées qui sont avancées par les réformateurs se fraient lentement un chemin et s'imposent peu à peu à travers la remise en cause des politiques traditionnelles caritatives (le modèle de la philanthropie privée : «à chacun ses pauvres 》) et la naissance de politiques sociales plus cohérentes et plus globales qui commencent à se dessiner dans le contexte de la crise économique et sociale de la fin des années $1880^{46}$.

L'Arrêté royal du 10 décembre 1881 , qui prévoit l'ouverture à la prison de Gand d'un quartier spécial de réforme pour jeunes délinquants, répond toujours aux mêmes besoins de séparer condamnés et acquittés. L'argument démographique reste la principale justification à cette création. Mais en même temps, le deuxième volet de cet arrêté témoigne déjà d'une percée des idées nouvelles qui préconisent, sur la base du constat d'échec des politiques pénitentiaires de moralisation, un nouveau type de classement, fondé cette fois sur des critères psychologiques et moraux : l'«incorrigibilité ». Ou, pour faire plus bref et plus clair : la lutte contre la récidive. L'Arrêté royal prévoit l'ouverture, à la prison de Gand, d'un quartier spécial pour «incorrigibles». C'est-à-dire les enfants que l'école de réforme n'a pu ni corriger, ni redresser, ni moraliser. Or ce quartier, dont la création est prévue dès 1881 , ne sera effectivement ouvert qu'en 1887 , soit un an après les événements de mars 1886.

Le contexte dans lequel s'opère cette innovation est évidemment tout à fait décisif : le pénal va de pair avec le social. Ce que n'a pu imposer le dernier gouvernement libéral en 1881 est réalisé par la nouvelle équipe catholique, six ans plus tard, sous le ministère de Jules Lejeune à la Justice, et sous la pression des besoins sociaux et de la nécessité d'une stratégie de maintien de l'ordre plus souple. L'idéologie de la défense sociale, miprotectrice, mi-répressive, s'exprime parfaitement dans l'ambigüité institutionnelle de ces créations: maison de réforme pour sauver les moins pervertis, quartier de discipline pour isoler les plus corrompus, sur lesquels, dit Lejeune, « la démoralisation a achevé son œuvre». Incorrigibles, irrécupérables qu'il faut traiter "comme de malheureux pestiférés ».

45. Enfants corrigés, enfants protégés. Analyse comparée des politiques de protection de l'enfance en Belgique, en France, aux Pays-Bas et au Québec (1820-1914), projet de recherche international en cours (1992-1994), cf. spécialement la rupture des années 1880 .

46. I. HENKINBRANT, La Naissance des politiques sociales en Belgique Le Débat au sein de la Commission pour la réforme de la bienfaisance (/895-1900), mémoire de licence en histoire, UCL, Louvain-la-Neuve,1994 (inédit). 
L'impact de cette création a, dans la pratique, fort peu d'effets sur le pénitencier de Saint-Hubert : la surpopulation y demeure constante et l'ouverture du quartier de Gand en 1881 ne se traduit pas par un abaissement du nombre des détenus à Saint-Hubert. Au contraire, dès décembre 1882, on atteint à nouveau la cote d'alerte (483 enfants). Sur le plan de la séparation des condamnés et des acquittés, aucun impact non plus. Même si la dénomination officielle change et que l'étiquette pénitentiaire de Saint-Hubert disparaît définitivement, puisque la maison est théoriquement réservée aux seuls acquittés, les populations n'en demeurent pas moins mélangées. Ce qui confirmera Lejeune dans son idée de supprimer cette distinction fondée sur le discernement, qui s'est révélée en pratique inapplicable.

Par contre, le quartier de discipline, ouvert à Gand en 1887 , obéit à une nouvelle logique de classification. Il est, selon les termes de l'Arrêté royal de création (21 mars 1887), réservé «aux natures absolument rebelles, déjouant toute tentative d'éducation, ainsi que les enfants dangereux par leurs mœurs et ceux qui auront donné des preuves constantes d'une perversité dans la maison de réforme $»$.

Le vocabulaire utilisé est particulièrement significatif des nouvelles théories criminologiques: les concepts de dangerosité et de «criminel d'habitude» - les récjdivistes qui «ont fait preuve d'une perversité constante" - ont remplacé le critère de discernement, donc de responsabilité et de faute. On est entré dans la logique de la défense sociale.

Les réformes de Jules Lejeune, en 1890, ne feront qu'entériner et confirmer cette nouvelle philosophie.

\section{4) Saint-Hubert: École de bienfaisance de l'État (1890-1921)}

Lorsque Jules Lejeune devient ministre de la Justice (1887) dans le gouvernement catholique de Beernaert, l'heure est aux réformes après la secousse sociale du printemps 1886 et les conclusions de la Commission du travail, instaurée par le gouvernement pour étudier les conditions de travail de la classe ouvrière et proposer des remèdes aux problèmes sociaux. La pression du tout jeune parti socialiste (Parti ouvrier belge, né en 1885), qui s'affirme comme le leader des opprimés en portant toutes les revendications sociales sur le terrain politique (conquête du pouvoir par le suffrage universel), agit fortement sur les catholiques «sociaux» qui commencent timidement à abandonner le libéralisme pur et dur pour se convertir à l'idée de l'intervention de l'État dans le champ social, à la suite du Congrès des Euvres sociales à Liège et surtout l'impact sur les catholiques belges de Rerum Novarum, 1891.

L'échec des libéraux classiques, la concurrence des socialistes qui arrivent au Parlement après les élections de 1894 (premières élections au suffrage universel, tempéré par la pluralité des voix accordée aux capacitaires, 
aux censitaires et aux pères de famille nombreuse) ont pour effet d'entraîner les catholiques dans un programme de réformes sociales qui démarre, timidement, en 1888 , avec une première loi sur la règlementation du travail des enfants employés dans les professions ambulantes et, en 1889, pour ceux qui travaillent dans les mines et industries (douze ans, douze heures). En 1889, on l'a vu, Jules Lejeune dépose son premier projet de loi sur la protection de l'enfance. Les socialistes se battent pour l'instruction primaire obligatoire. Si ces lois de protection doivent attendre plus de vingt ans pour être votées (1912 et 1914), le débat sur l'enfance est largement entamé dès la fin des années 1880 .

C'est dans ce contexte qu'il faut situer l'Arrêté royal du 9 juillet 1890 qui réorganise tout le système pénitentiaire pour les jeunes délinquants et vagabonds. Le processus qui mène à cette réforme et les moyens tactiques utilisés par Lejeune pour faire passer ses idées en l'absence de toute décision parlementaire repose sur une campagne de mobilisation générale de l'opinion : organisation de Congrès internationaux sur la protection de l'enfance à Anvers en 1890,1894 et 1898, création de sociétés protectrices et de comités de patronage, politique de promotion des idées nouvelles dans le milieu des juristes par le biais d'innombrables brochures spécialisées consacrées à la protection de l'enfance, aux tribunaux pour enfants, diffusion de ces campagnes dans le public par le relais de la presse qui ouvre largement ses colonnes à tous les philanthropes soucieux de faire connaître leur action et de recueillir des fonds ${ }^{47}$...

Lejeune, qui est l'âme incontestable et incontestée de ce mouvement, l'homme-orchestre, présent sur tous les fronts, insuffle à ses fidèles l'énergie combative en même temps que, porté par eux, il parvient à réaliser, par étapes, son programme de réformes : condamnation et libération conditionnelles (loi Lejeune de 1888), loi sur le vagabondage et la mendicité (1891), création des Écoles de bienfaisance (1890). Celles-ci sont destinées à remplacer tous les anciens pénitenciers et écoles de réforme par un type unique d'institutions centrées, comme leur nom l'indique, sur la bienfaisance et non plus la prison, la punition, la réforme.

L'Arrêté de 1890 rompt une fois pour toutes, en théorie du moins, avec la vision pénitentiaire fondée sur la notion de culpabilité. L'enfance en danger succède à l'enfance coupable. Elle devient la cible privilégiée de la bienfaisance ; l'objectif est désormais d'éduquer, par l'école, et non plus de

47. Sur cette campagne, cf. notamment : M.-S. DUPONT-BOUCHAT, «La Belgique capitale intemationale du patronage (1894-1914)", in Commission royale des patronages (1894-1994) (sous presse); A. M. TEIRLYNCK, « La Commission Royale des Patronages (1894-1994)", Ibid., F. HUART, La Société protectrice des enfants martyrs de Bruxelles (1892-1912), mémoire de licence en histoire, UCL, 2 vol., Louvain-la-Neuve, 1994 (inédit). 
punir, par la prison, ni même de réformer par la moralisation. Et c'est l'État qui doit remplir cette mission éducative. L'appellation d'École de bienfaisance de l'État exprime cette nouvelle orientation et cette volonté de rupture, amorcées, on l'a vu, dès les années 1880 .

Comment ces changements de politique se sont-ils traduits dans la pratique des institutions? Quel est l'impact de cette réforme radicale du sytème pénitentiaire pour les jeunes délinquants? Quel est l'effet de ce nouveau changement d'appellation et d'orientation sur le terrain et plus particulièrement ici, pour le pénitencier de Saint-Hubert?

Entre 1891 et 1912 se mettent progressivement en place les structures qui doivent permettre la réalisation de cette nouvelle politique d'éducation et de protection.

L'optimisme des nouveaux réformateurs n'a rien à envier à celui des pères fondateurs. Si l'on en croit $A$. Van Waesberghe, qui fut directeur de l'école de bienfaisance de Saint-Hubert de 1906 à 1911, avant de devenir ensuite directeur de l'école de bienfaisance de Ruiselede-Beernem, le mot d'ordre est désormais : "Tout à l'éducation et à la protection. 》 Il publie en 1911 un ouvrage sur Un système familial d'éducation pénitentiaire ${ }^{48} \mathrm{et}$, en 1914, un article sur les Écoles de bienfaisance ${ }^{49}$ où il fait longuement référence à son expérience personnelle. Voici comment il présente la réforme :

On remédie aux classifications mal faites [...] On se montre de plus en plus sévère pour la désignation des membres du personnel qui souvent, surtout les surveillants, étaient puisés dans les couches les moins intéressantes de la population ( $\mathrm{sic}$ )... On applique les méthodes les plus modemes de la pédagogie à l'instruction tant professionnelle que scolaire. L'étude et la séparation des anormaux est introduite; les dernières découvertes pratiques de la pédagogie sont appliquées. Les méthodes de placement des élèves, l'organisation des comités de patronage sont mises au point. Tout est à l'éducation et à la protection; le principe de la répression n'est plus mis en pratique dans les écoles de bienfaisance ${ }^{50}$.

Reste à voir si ce bel optimisme correspond à un véritable changement dans la réalité quotidienne de la pratique des écoles de bienfaisance et, tout d'abord, comment les mesures prévues «sur le papier» vont passer dans l'organisation des établissements.

48. A. VAN WAESBERGHE, «Un système familial d'éducation pénitentiaire», in Revue de droit pénal et de criminologie, mai 1991.

49. A. VAN WAESBERGHE, "Les écoles de bienfaisance", in CAMPIONO, L'État et l'enfant, Bnuxelles, 1914, pp. 442-497.

50. Ibid., pp. $452-453$ 
Sur le plan institutionnel, déjà, la tâche s'avère compliquée. Dès 1893 , un service spécial est créé à la quatrième division du ministère de la Justice pour s'occuper des questions en souffrance depuis quatre ans et auxquelles il est urgent de répondre.

Il faut confectionner un nouveau règlement pour unifier le régime des différentes institutions désormais réunies sous le vocable unique d'Écoles de bienfaisance. Il faut choisir un nouveau modèle de discipline qui soit à la fois compatible avec les nouveaux principes de la réforme - tout à l'éducation, fin de la répression - et les exigences de maintien de l'ordre intérieur, et il faut trouver une solution pour les incorrigibles qui demeurent exclus du bénéfice de cette réforme. Il faut effectuer un nouveau tri entre les enfants «en danger " qu'il s'agit de protéger et les enfants dangereux à isoler pour éviter qu'ils ne contaminent les autres au sein d'institutions désormais entièrement vouées à l'éducation plutôt qu'au redressement. Le quartier des incorrigibles à Gand continuera à fonctionner après la réforme de Lejeune. Il en constitue d'ailleurs le volet répressif qui vient contrebalancer l'assouplissement concédé dans le régime des nouvelles écoles.

C'est une illustration parfaite de la politique de défense sociale qui vise à protéger les «bons»- les enfants en danger - et à punir plus sévèrement les «méchants »- les incorrigibles, les réciđivistes.

La mise en application des principes de Lejeune reste cependant bien délicate et le ministre lui-même ne paraît pas toujours avoir une idée très claire de la différence de discipline qu'il faut instaurer. Il écrit, dans une note du 7 août 1893 :

Aurons-nous des écoles à régime doux et à régìme sévère? En quoi consistera la différence entre régime sévère et régime doux et surtout entre régime sévère et discipline? Quels seront les établissements affectés à telles catégories d'enfants ${ }^{51}$ ?

Il impute le retard dans la mise en œuvre des réformes à des raisons matérielles d'abord: l'insuffisance des locaux, vieux problème toujours récurrent et jamais résolu. Mais aussi à l'impossibilité de réaliser dans la pratique l'organisation créée sur le papier. Il faudra attendre 1906 pour que soit enfin élaboré le nouveau règlement des écoles de bienfaisance, soit seize ans après leur création.

Entre-temps, des dizaines de circulaires ministérielles viennent préciser, point par point et mois après mois, les détails de la nouvelle organisation. Il serait trop long et fastidieux d'en énumérer ici les étapes, et l'on

51. Archives générales du Royaume à Bruxelles, Ministère de la Justice, no 99. lettre de Jules Lejeune au directeur de Saint-Hubert, 7 août 1893. 
peut se borner à ne reprendre que les dispositions les plus marquantes qui éclairent l'esprit de la réforme.

L'article 2 de la loi du 27 novembre 1891 pour la répression du vagabondage et de la mendicité stipule que :

Les écoles de bienfaisance seront affectées aux individus âgés de moins de dix-huit ans accomplis qui seront mis par l'autorité judiciaire à la disposition du gouvernement et dont l'admission aura été demandée par les autorités communales.

Voilà pour les vagabonds, désormais assimilés aux délinquants et mis à la disposition du gouvernement, qu'il faut protéger «dans leur intérêt». C'est aussi un moyen pour les communes de se débarrasser de la charge de leur entretien sur l'État.

Les articles 24 à 33 de la même loi précisent que les enfants qui n'auront pas atteint l'âge de treize ans accomplis à la date de leur entrée dans une école de bienfaisance resteront pendant toute la durée de leur internement totalement séparés des individus entrés à un âge plus avancé. Il en ira de même pour la catégorie des treize-seize ans. En principe, tous demeureront à l'école de bienfaisance jusqu'à l'âge de leur majorité, mais ils pourront être placés en apprentissage chez un cultivateur ou un artisan; ils pourront également être rendus conditionnellement à leurs parents, si ceux-ci présentent toutes les garanties de moralité et sont aptes à les surveiller. Mais si ce n'est pas le cas, ils devront réintégrer l'école de bienfaisance.

L'objectif avoué de cette loi, qui s'inscrit parfaitement dans la logique de la politique de défense sociale, est de se montrer plus sévère à l'égard des «fainéants d'habitude» en prévenant la récidive :

La multitude des récidivistes qui reprennent sans cesse le chemin des dépôts de mendicité montre clairement que le régime adopté n'est pas assez répressif [...] Il importe à tous points de vue de soumettre à un régime plus sévère et plus répressif les mendiants et vagabonds vicieux qui ne veulent pas travailler et de traiter avec plus d'humanité et de prévoyance les invalides et les malheureux qui ne peuvent pas travailler 52 .

En ce qui concerne les mineurs, il s'agit, toujours selon Lejeune, de

supprimer les promiscuités pernicieuses et surtout de préserver de la contagion du mal les jeunes gens et les enfants. On espère ainsi arracher un grand nombre de détenus aux funestes traditions de la paresse, de l'ignorance et du vice.

52. Pasinomie, loi du 27 novembre 1891, p. 445, exposé des motifs signé par J. Lejeune. 
D'où l'allongement de la durée de l'internement... Ce qui prouve, si besoin en était, qu'il ne s'agit pas uniquement de protection, mais que, sous le couvert d'un nouveau vocabulaire, la répression conserve tous ses droits.

En fait, la loi de 1891 aboutit, sous prétexte de protection, à élargir considérablement le champ de la répression en utilisant des mesures d'ordre administratif pour interner des catégories de plus en plus larges d'individus, considérés comme «dangereux» pour l'ordre public. Ces mesures sont considérées comme étant « d'intérêt général et de sécurité publique dont la charge incombe à l'État ${ }^{53}$ ».

Pour appliquer cette nouvelle stratégie d'encadrement et de maintien de l'ordre, il faut multiplier les établissements car les institutions existantes - pénitenciers, écoles de réforme - ne peuvent suffire à accueillir les catégories de plus en plus larges de jeunes délinquants, vagabonds ou mendiants, désormais tous confondus dans le public des écoles de bienfaisance.

Une nouvelle école de bienfaisance est créée à Mol le 29 janvier1894, une autre à Ypres, le 28 juillet de la même année. Pour les filles, un quartier spécial de discipline est ouvert à la maison de refuge de Bruges (27 avril 1894) pour y transférer les élèves dont la présence dans une école de bienfaisance serait jugée dangereuse pour les autres internées ${ }^{54}$. En clair, ceci vise les filles-mères et les jeunes prostituées dont le mauvais exemple est toujours à craindre.

La succursale de Saint-Hubert à Namur qui fonctionnait depuis 1871 est désaffectée pour les garçons à partir du 18 juin 1894 ; les élèves seront renvoyés à Saint-Hubert ou dirigés vers la nouvelle école de Mol, en fonction de critèr es de répartition des populations pénitentiaires selon l'âge et l'origine géographique.

Les circulaires qui règlent cette répartition se succèdent en s'efforçant de préciser des critères «rationnels » qui combinent les impératifs d'ordre législatif avec ceux de l'âge et de l'origine géographique des enfants. Cela aboutit à un système particulièrement complexe qui n'est pas plus facile à mettre en œuvre que les clés de répartition anciennement adoptées et vivement critiquées... La logique de l'administration pénitentiaire demeure une énigme difficile à résoudre en pratique.

D'après la circulaire du 21 mai 1896, l'école de bienfaisance de SaintHubert sera affectée aux garçons de moins de seize ans, internés en vertu

53. Ibid., p. 453, rapport fait à la Chambre des représentants, au nom de la Commission, par A. Visart.

54. A. DELACOLLETTE, Contribution à l'histoire de la protection de l'enfance en Belgique, Merksplas, 1949, première partie, p. 55 : quartier de discipline des écoles de bienfaisance pour filles. 
des articles 25 et 26 de la loi de 1891 , et des articles du 72 et 76 du code pénal. Y seront envoyés les enfants des neuf provinces âgés de onze à quatorze ans et ceux de quatorze à dix-huit ans originaires des provinces de Brabant, Flandre orientale, Liège et Luxembourg. Les garçons des autres provinces seront répartis entre Mol et Reckheim; les moins de onze ans iront à Ruiselede. Les filles de moins de treize seront internées à Beernem, et les autres à Namur...

On conçoit que l'application de ces règles ne manquera pas de susciter quelques difficultés.

Ce classement sera simplifié en 1904, suite à la révision de la loi de 1891 , en 1897, en ce qui concerne les mineurs. On ne retiendra dorénavant que le seul critère de l'âge : les garçons de moins de treize ans iront à Ruiselede, ceux de treize à seize ans seront répartis entre Mol et Ypres et ceux de seize à vingt et un ans seront dirigés sur Saint-Hubert. Pour les filles, pas de changement : les petites à Beernem et les grandes à Namur ${ }^{55}$.

La circulaire ministérielle du 24 novembre 1904 règle en même temps les problèmes de discipline : un quartier spécial sera annexé à la prison centrale de Gand pour les garçons et à l'école de bienfaisance de Namur pour les filles. Le ministre de la Justice pourra y faire transférer les élèves qui seront jugés «vicieux, incorrigibles ou qui exigent une surveillance spéciale».

Un quartier spécial est également créé à Ypres pour les garçons réintégrés à la suite d'une faute grave commise après leur libération conditionnelle ou leur mise en apprentissage ${ }^{56}$.

Voilà donc traduits dans l'organisation institutionnelle les principes de l'Arrêté royal de 1890 et des lois de 1891 et 1897 qui visaient à séparer les enfants «en danger » des enfants «dangereux».

Le règlement gênéral uniformisant le régime intérieur des écoles de bienfaisance, promulgué le 9 janvier 1906, vient couronner ce nouvel édifice en mettant le point final à ce que Lejeune appelait la réorganisation «sur papier».

Par rapport au premier règlement de Saint-Hubert (1847), le nouveau règlement des écoles de bienfaisance apporte des modifications importantes qui témoignent du changement d'esprit qui s'est opéré en un demi-siècle en ce qui concerne la réducation et le redressement.

À l'époque où furent créés le pénitencier de Saint-Hubert (1840) et les premières écoles de réforme (Ruiselede et Beernem, 1848), les idées de

55. Ibid., pp. 58-60.

56. Sur cette répartition, cf. le tableau en annexe : Institutions pénitentiaires pour jeunes délinquants en Belgique : destination/population (1832-1912). 
moralisation chères à Ducpétiaux s'imposaient. Moralisation par la religion, par le travail, par la discipline, par la classification des élèves selon leurs mérites entre les sections de punition, d'épreuve et de récompense, avec citation au tableau d'honneur pour les plus méritants.

Si cette classification est reprise telle quelle en 1906 (articles 169 et $170 \mathrm{du}$ règlement), si les devoirs des élèves demeurent inchangés — « respect scrupuleux de la discipline, de l'ordre, de la propreté et de l'hygiène »-l'accent est mis désormais sur la condition physique, la formation professionnelle, le placement en apprentissage, l'épargne.

La différence la plus marquante par rapport aux périodes antérieures se situe dans la médicalisation des jeunes détenus. Celle-ci débute à Saint-Hubert avec la création, le 6 mars 1913, d'un quartier spécial d'observation pour examen des cas urgents d'anomalie. Le 30 décembre de la même année, l'établissement central d'observation est transféré à Mol et la direction en est confiée à Maurice Rouvroy, qui devient sous-directeur de Mol en 1913, puis directeur, en 1919. En novembre 1912, alors qu'il n'était encore qu'instituteur à Saint-Hubert, M. Rouvroy avait été chargé de s'initier théoriquement et pratiquement à l'observation des mineurs comparaissant devant la justice. Il avait rédigé un rapport basé sur son expérience personnelle, à partir des observations qu'il avait réalisées sur les élèves de SaintHubert, qu'il concluait déjà en ces termes:

Le temps n'est plus où l'on se contentait, en éducation, des procédés d'introspection, d'observation de soi-même, où l'on se contentait d'assimiler la mentalité de l'enfant à celle de l'homme fait. Les différences essentielles que l'on constate entre l'âme juvénile et l'âme adulte, en même temps que la diversité des caractères individuels, exigent que l'on fasse appel à la psychologie objective, à l'étude attentive des mentalités ${ }^{57}$.

En février et mars 1913, il visite diverses institutions pour enfants arriérés, notamment à Gand et Charleroi, et l'École normale de Carlsbourg. Il conclut son rapport sur les techniques et l'outillage d'observation mis en auvre dans ces institutions en affirmant que le meilleur outil demeure la bonté du maître, la confiance, la politique du «çeur à cœur » qui assurent le succès de l'observation des caractères.

M. Rouvroy sera le pionnier de ce système d'observation qui va bientôt faire école et qui sera célébré par la suite comme une des réussites les plus notables du traitement et de la sériation des jeunes détenus. Celle-ci modernise en quelque sorte la vieille idée đe Ducpétiaux qui avait instauré la tenue d'un livre de la comptabilité morale destiné à enregistrer tous les progrès (et les méfaits) des jeunes détenus.

57. Archives de l'État à Saint-Hubert, École de bienfaisance, rapport signé par M. Rouvroy, 12 juin 1912. 
Si le terme de "comptabilité morale » est toujours utilisé par Rouvroy, il en modernise le principe et le contenu : la comptabilité morale devient la science des caractères. C'est dans son «laboratoire» de l'école de bienfaisance de Saint-Hubert qu'il commence à élaborer une véritable science de l'enfant qui vaudra au centre d'observation de Mol et à son directeur une réputation internationale ${ }^{58}$.

Dans les années 1930, lorsque les grands scandales éclatent dans les pénitenciers français, le journaliste Alexis Danan entreprend une campagne de presse et publie, en 1936, un ouvrage retentissant intitule Maisons de supplices. Il consacre la première partie aux bagnes d'enfants français qui ont pour nom Saint-Maurice, Saint-Hilaire, Belle-Île-en-Mer, Aniane et Mettray ${ }^{59}$. En contrepoint, dans la seconde partie, il évoque les maisons belges, qui lui apparaissent comme de véritables paradis. Il y rent un vibrant hommage à Maurice Rouvroy :

Je n'ai jamais vu, je crois, depuis que je m'impose à la patience des pédiatres, de savant plus informé des choses de sa science, mais surtout plus décidé que celui-ci à les envisager sous l'angle de l'humanité courante [...] Quand on se demande ce qu'on admire le plus chez ce psychologue d'une lucidité quasi surhumaine, on en vient à découvrir que c'est l'homme et les infinies ressources de son cœur ${ }^{60}$.

Ses louanges vont aussi au directeur de l'école de bienfaisance de Saint-Hubert, M. Legrain, qu'il dépeint comme « Un brave homme, un vrai père de famille, bon et miséricordieux pour ses élèves. Il prône la patience et la bonté et se montre paternel vis-à-vis de ces enfants déshérités, difficiles, certes, mais avides d'affection ».

Bref, si l'on en croit A. Danan, la Belgique, et plus particulièrement l'école de Saint-Hubert, représente le modèle parfait, le système le plus humain, le mieux géré, et ce par des maîtres compétents et remarquables qu'il offre en exemple à tous les éducateurs français :

Mettons-nous bravement à l'école des meilleurs, imitant les Belges qui depuis quinze ans n'ont plus de bagnes d'enfants, sans avoir plus que nous - et il s'en faut - de récidivistes, de révoltés, de criminels ${ }^{61}$.

58. Sur l'œuvre de Maurice Rouvroy, cf. M. D'HOKER, "Contribution de Maurice Rouvroy (1879-1974) aux soins en résidence de la jeunesse a problèmes psycho-sociaux pendant l'entre-deux-guerres ", Beyond the Pale, behind Bars, Marginalization and Institutionalization from the 18th to the 20th Century, Paedagogica Historica, numéro spécial, t. 26, 1990, pp. 211-222.

59. Sur les maisons de correction françaises, cf. H. GAlLLAC, Les Maisons de correction (1830-1945), $2^{e}$ édition, Paris, Cujas, 1991.

60. A DANAN, Maisons de supplices, Paris, Denoèl, 1936, p. 186.

61. A DANAN, «Quand les bagnes d'enfants seront supprimés, par quoi les remplacera-t-on? ", Paris-Soir, 22 novembre 1934. 
L'insistance avec laquelle Danan oppose le modèle belge, paré de toutes les vertus, au modèle français, exécrable, ne doit cependant pas nous abuser : il mène campagne pour la fermeture de Mettray et les articles qu'il publie dans Paris-Soir en 1934 ont des titres éloquents: "Une honte: Mettray, établissement privé de tortures ", «Dans cette maison paternelle, on supplicie les malheureux gosses placés en éducation surveillée», "Des instituteurs jllettrés», "Des enfants à la torture », et pour finir : «Il faut fermer Mettray».

S'il avait interrogé à la même époque des ex-détenus de Saint-Hubert, aurait-il recueilli les mêmes témoignages accablants?

À partir de quand la situation commença-t-elle à se dégrader? Le scandale qui aboutit à la fermeture du pénitencier en 1954 offre des similitudes avec celui des maisons de correction françaises en 1934. Mais à cette époque, Saint-Hubert apparaissait encore, aux yeux de l'opinion extérieure au moins, comme un "pénitencier modèle »...

N.D.R. Dans la seconde partie, le lecteur trouvera le développement des points suivants :

2. La succursale du pénitencier de Saint-Hubert à Namur (1871-1896)

3. Le pénitencier des filles à Namur (1864-1890) 